\title{
TESTE DE ACIDEZ GRAXA COMO ÍNDICE DE QUALIDADE EM ARROZ
}

\author{
Evaluation of fat acidity test as rice quality index
}

\author{
Marco Antônio Martin Biaggioni ${ }^{1}$, Roberta Espíndola Barros ${ }^{2}$
}

\begin{abstract}
RESUMO
Pesquisas visando a redução de perdas de grãos e sementes durante o armazenamento têm ocupado destaque em vários países. Dentro deste contexto, a avaliação da eficácia de um índice de qualidade de boa aplicabilidade, com metodologia simples e de resposta imediata visando tomadas rápidas de decisão é de suma importância. O presente trabalho, conduzido no Laboratório de Processamento de Produtos Agrícolas - UNESP, Botucatu/SP teve como objetivos: (a) estabelecer correspondência entre o nível de ácidos graxos livres e as classes de vigor em sementes; (b) estabelecer correspondência entre o nível de ácidos graxos livres e a classificação comercial por tipos, em grãos de arroz (Oryza sativa L.). A correspondência entre o nível de ácidos livres e as classes de vigor em sementes foi avaliada utilizando-se sementes envelhecidas artificialmente, obtendo-se assim níveis diferenciados de vigor. A correspondência entre o nível de ácidos graxos livres e a classificação por tipos, em grãos de arroz, foi realizada utilizando-se amostras de arroz com as porcentagens máximas de grãos "defeituosos" permitidos pela legislação vigente. Utilizou-se a análise de variância de um delineamento inteiramente ao acaso e, para comparação entre médias, aplicou-se o teste de Tukey ao nível de 5\% de probabilidade. Por meio dos resultados obtidos, observou-se que o teste de acidez graxa mostrou-se exequível para avaliar o vigor de sementes de arroz. Na pesquisa que buscava uma correspondência entre os valores de acidez graxa e classificação comercial, os dados revelaram a tendência do nível de ácidos graxos livres acompanhar a classificação comercial por tipos.
\end{abstract}

Termos para indexação: Ácidos graxos livres, qualidade de semente, classificação arroz, Oryza sativa .

\begin{abstract}
Researches aiming reduction of grain and seed losses during storage period has been developed in many countries. Quality grain indexes of good applicability with simple methodology to give quick response for quick taking decision are of importance. The present study was carried out at the Agricultural Products Processing Laboratory - UNESP, Botucatu/SP, with the objectives of: a) establishing a relation between the levels of free fatty acids and the seeds vigor classes; $b$ ) establishing the relation between the level of free fatty acids and commercial classification through types in rice grains (Oryza sativa L.). The correspondence between the free fatty acids and seeds vigor classes was assessed by using seeds artificially degenerated, obtaining different vigor rates. The relation between the level of free fatty acids and classification through types in rice grains was done by using samples of rice with the maximum percentage of "blemished" grains allowed by the legislation in force. It was used the variance analysis in a completely randomized blockdesign and, for means comparison, it was used the Tukey's test at 5\% of probability. In seeds, the fat acidity test showed to be able to evaluate the rice seeds vigor. The results from the study searching for a relation between the values of fat acidity and commercial classification revealed a tendency of the free fatty acids rate to follow the commercial classification through types.
\end{abstract}

Index terms: Free fat acids, seed quality, rice classification, Oryza sativa.

(Recebido para publicação em 22 de novembro de 2004 e aprovado em 7 de fevereiro de 2006)

\section{INTRODUÇÃO}

\section{Qualidade de sementes de arroz}

Segundo Krzyzanowski et al. (1999), o teste de germinação é considerado padronizado, com ampla possibilidade de repetição dos resultados, dentro de níveis razoáveis de tolerância, desde que sejam seguidas as instruções estabelecidas em Regras para Análises de Sementes, tanto nacionais como internacionais. Ainda que os resultados de testes de germinação apresentam alto grau de confiabilidade, sob o aspecto de reprodutibilidade dos resultados e possibilidade de utilização como base para a fiscalização do comércio, o mesmo não ocorre quando se trata da utilização de lotes para a semeadura em campo, onde, com grande frequiência, os resultados de emergência das plântulas podem ser consideravelmente inferiores aos observados para a germinação em laboratório.

De modo geral, os testes de vigor permitem uma avaliação mais sensível da qualidade da semente, em relação à germinação, fornecendo uma classificação mais consistente de lotes, com qualidades distintas em relação ao seu potencial de desempenho em campo e/ou armazenamento (RIBEIRO, 1999). Entre os testes de vigor utilizados para sementes de arroz, pode-se destacar aqueles que buscam um indicativo da deterioração no seu estágio mais incipiente, como o teste de condutividade elétrica

${ }_{1}^{1}$ Professor Assistente, Doutor, Departamento Engenharia Rural - Faculdade de Ciências Agronômicas/UNESP - Botucatu,SP.

${ }^{2}$ Mestre em Agronomia, Área de Concentração Energia na Agricultura - Departamento Engenharia Rural, F.C.A. /UNESP - Botucatu, SP. 
que procura quantificar a perda de integridade das membranas celulares. $\mathrm{O}$ teste de envelhecimento acelerado, por sua vez, propõe-se estimar o potencial de armazenamento dos lotes processados, expondo-os a condições adversas de temperatura e umidade relativa do ar.

O teste de acidez graxa, como método para avaliar a deterioração em grãos armazenados, tem na sua sensibilidade a principal vantagem. Como a formação de ácidos graxos livres nos grãos é resultante da hidrólise das gorduras, esta análise permite, além da quantificação do processo deteriorativo, acusá-lo ainda nos estágios iniciais. O bom poder de resposta deste método, associado à sua rapidez e baixo custo na execução tem suscitado investigações de caráter mais aplicado, visando um melhor aproveitamento do teste na área de colheita e processamento de grãos e sementes, com possibilidades de integrar, de forma efetiva, um conjunto de análises de rotina.

Modificações expressivas nas principais reservas ocorrem quando as sementes se deterioram. Uma das alterações associadas com a deterioração de sementes, em geral, é a sua acidificação (ABDUL-BAKI $\&$ ANDERSON, 1972). Em estudos tem sido observado que essa acidificação é o resultado do aumento de ácidos graxos livres, de fosfatos ácidos e de aminoácidos, produzidos pela ação das lipases, fitases e proteases, respectivamente. Entre esses três grupos de compostos, o maior e o mais rápido aumento ocorre nos ácidos graxos (SMITH \& BERJAK, 1995, citados por PEREIRA, 1999).

Soares (2003), avaliando o nível de ácidos graxos livres em grãos de soja danificados artificialmente, concluiu que o teste de acidez graxa mostrou-se sensível aos efeitos dos tratamentos de dano térmico e mecânico, em relação à testemunha e, que, comparado ao teste de tetrazólio, o índice de acidez graxa revelou-se num teste mais poderoso para detectar o efeito latente oriundo de danos térmico e mecânico. O mesmo autor ainda afirma que o teste de acidez graxa mostrou-se mais sensível em relação aos testes de qualidade industrial aplicados à soja, confirmando a significativa deterioração ocorrida durante o armazenamento, não detectada, porém, pelas análises de teor de óleo e rancidez.

Em sementes de trigo, Yanagiwara et al. (2004) concluíram que com a análise de acidez graxa, como teste de vigor, foi possível detectar uma discreta perda de qualidade durante sete meses de armazenamento, correlacionado-se significativamente, apenas, com o teste de condutividade elétrica. $O$ teste de vigor "peso de matéria seca" foi considerado o mais sensível e o de emergência em campo o menos sensível. Nessa mesma pesquisa, o teste de acidez graxa também mostrou-se sensível durante a avaliação do vigor das sementes.

A rápida obtenção de resultados vem assumindo, cada vez mais, destaque nas etapas de produção de semente, pois, além de agilizar o processo de produção, permite a redução dos custos (MARCOS FILHO et al., 1987). Sob esse aspecto, o teste de ácidos graxos livres demonstra ser de grande interesse, pois permite detectar a fase inicial do processo degenerativo, possibilitando tomadas rápidas de decisão, com o intuito de reduzir ou minimizar o seu efeito sobre a qualidade fisiológica das sementes.

\section{Classificação comercial dos grãos arroz}

Além da variação da acidez graxa com a qualidade das sementes, pode existir uma relação entre o nível de ácidos graxos livres e a classificação comercial por tipos, em grãos de arroz, cujo estudo passa a ser de grande importância.

De acordo com a Portaria $\mathrm{n}_{-}^{\circ} 269$ de 17 de novembro de 1988, Decreto $\mathrm{n}_{-}^{\circ} 82.110$, de 14 de agosto de 1978, do Ministério da Agricultura, o arroz é classificado em grupos, subgrupos, classes e tipos. Segundo a sua forma de apresentação, o arroz é classificado em dois grupos, assim denominados: arroz em casca e arroz beneficiado. Segundo o seu preparo, o arroz em casca e o arroz beneficiado serão ordenados em subgrupos, sendo estes natural e parboilizado (subgrupos do arroz em casca) e integral, parboilizado, parboilizado integral e polido (subgrupos do arroz beneficiado). $\mathrm{O}$ arroz em casca e o arroz beneficiado serão divididos em cinco classes, assim identificadas: longo fino, longo, médio, curto e misturado. Qualquer que seja o grupo e o subgrupo a que pertença, o arroz será classificado em cinco tipos, expressos por números de 1 a 5 , e definidos pelo percentual de ocorrência de defeitos graves, defeitos gerais agregados ou de grãos quebrados e quirera.

Ainda segundo a portaria, é considerado defeito grave a presença de matérias estranhas, impurezas, grãos mofados, ardidos, pretos e não gelatinizados. Defeitos gerais agregados é o somatório dos defeitos gerais encontrados na amostra, sendo considerado defeitos gerais os grãos danificados, manchados, picados, amarelos, rajados, gessados e não parboilizados.

Tendo em vista a possibilidade de adoção de um índice de qualidade altamente sensível à deterioração, com metodologia simples, rápida e de boa aplicabilidade para

Ciênc. agrotec., Lavras, v. 30, n. 4, p. 679-684, jul./ago., 2006 
grãos e sementes de arroz (Oryza sativa L.), o presente trabalho teve como objetivos principais:

- estabelecer correspondência entre o nível de ácidos graxos livres e as classes de "vigor", em sementes; - estabelecer correspondência entre o nível de ácidos graxos livres e "tipos", em grãos de arroz (Classificação comercial).

\section{MATERIALE MÉTODOS}

\section{Acidez graxa e teste de vigor em sementes de arroz}

Foram utilizadas sementes de arroz da cultivar IAC-102, lote TB-24/02, oriundas da Coordenadoria de Assistência Técnica Integral (CATI/Avaré). A aquisição de sementes com diferentes níveis de qualidade é de difícil viabilização, tendo em vista a tendência das empresas do ramo manterem em seus estoques apenas material de elevada qualidade. Dessa forma, sementes com diferentes níveis de qualidade foram obtidas por meio do teste de envelhecimento artificial, no Laboratório de Análises de Sementes do departamento de Produção Vegetal da UNESP/Botucatu. As sementes foram acondicionadas em caixas tipo "Gerbox", com 40 $\mathrm{mL}$ de água destilada, e em seguida no germinador, a $42^{\circ} \mathrm{C}$ e $100 \%$ de umidade relativa, onde permaneceram por períodos de 0, 72, 96, 144 e 192 horas. Através desse processo foram diferenciados cinco sub-lotes de sementes com vigor de $78 \% ; 37,5 \% ; 34 \% ; 18,5 \%$ e $11,5 \%$. Após homogeneização, amostras formadas por quatro repetições de cinqüenta sementes foram submetidas às análises de condutividade elétrica (VIEIRA, 1994), envelhecimento acelerado (VIEIRA, 1994) e teste padrão de germinação (BRASIL, 1992). Para análise de acidez graxa, foram utilizadas amostras de 40 gramas, em quatro repetições, conforme AACC (1995).

\section{Acidez graxa e classificação comercial em grãos de arroz}

Para o estudo da relação entre o nível de ácidos graxos livres e a classificação por tipos, em grãos de arroz, cinco lotes de grãos, com qualidade variável, provenientes da Cerealista São João, localizada em Santa Cruz do Rio Pardo/SP, foram submetidos à classificação física no próprio laboratório da empresa, seguida das análises de acidez graxa realizadas no Laboratório de Processamento de Produtos Agrícolas da FCA/UNESPBotucatu.

O critério de avaliação adotado no presente trabalho, como referência para verificar a correspondência entre o nível de ácidos graxos livres e a classificação comercial de grãos de arroz, foi determinar o nível de acidez graxa para cada tipo de arroz no limite máximo estabelecido para cada defeito (geral e agregado). Assim, visou-se determinar o nível de acidez graxa limitante para cada tipo de arroz. Para tanto, foram preparadas as amostras de $40 \mathrm{~g}$ necessárias a cada repetição da análise de acidez graxa com as porcentagens máximas de grãos "defeituosos" (Quadro 1). Este procedimento foi adotado com o intuito de evitar falhas na representatividade das amostras, já que a quantidade, em gramas, de grãos permitidos com defeitos graves é mínima e, possivelmente, ao retirar amostras para realizar a análise, esta poderia não conter a quantidade exata de cada defeito.

QUADRO 1 - Arroz beneficiado polido (limites máximo de tolerância de defeitos/ tipos/g/ amostra de $40 \mathrm{~g}$ ).

\begin{tabular}{|c|c|c|c|c|c|}
\hline \multirow[b]{2}{*}{ Tipo } & \multicolumn{2}{|c|}{ Defeitos Graves } & \multirow[b]{2}{*}{$\begin{array}{c}\text { Defeitos } \\
\text { Agregados }\end{array}$} & \multirow[b]{2}{*}{$\begin{array}{c}\text { Total de } \\
\text { Quebra-dos e } \\
\text { Quirera }\end{array}$} & \multirow[b]{2}{*}{$\begin{array}{l}\text { Quirera } \\
\text { (Máximo) }\end{array}$} \\
\hline & $\begin{array}{l}\text { Matéria estranhas } \\
\text { e impurezas }\end{array}$ & $\begin{array}{l}\text { Mofados e } \\
\text { ardidos }\end{array}$ & & & \\
\hline 1 & 0,10 & 0,10 & 1,60 & 4,00 & 0,20 \\
\hline 2 & 0,20 & 0,20 & 3,20 & 8,00 & 0,40 \\
\hline 3 & 0,40 & 0,40 & 5,60 & 12,00 & 0,80 \\
\hline 4 & 0,60 & 0,80 & 8,80 & 16,00 & 1,20 \\
\hline 5 & 0,80 & 1,60 & 13,60 & 20,00 & 1,60 \\
\hline
\end{tabular}




\section{Análise estatística}

Para a análise estatística do vigor de sementes com diferentes níveis de deterioração, foi adotado o delineamento inteiramente casualizado, para os testes de vigor condutividade elétrica, germinação, envelhecimento acelerado e acidez graxa. Foram utilizadas quatro repetições e aplicado o teste de Tukey no nível de 5\% de significância para comparação das médias. Também foi realizada correlação entre o teste de acidez graxa e os demais testes de qualidade.

Para a análise da classificação comercial de grãos foi adotado o mesmo procedimento estatístico descrito acima, formado pelos tipos de arroz (1,2,3,4 e 5) e o nível de acidez graxa.

\section{RESULTADOS E DISCUSSÃO}

Acidez graxa e as classes de "vigor" em sementes de arroz

Os resultados da análise estatística dos testes utilizados para avaliação da qualidade em sementes envelhecidas artificialmente são apresentados no Quadro 2. Para melhor entendimento, foi denominado de lotes 1, 2, 3, 4 e 5 as sementes submetidas ao envelhecimento artificial por 0, 72, 96, 120 e 192 horas, respectivamente.

Pode-se observar que a metodologia utilizada no envelhecimento artificial realizado nas sementes com a finalidade de se adquirir lotes diferenciados quanto ao vigor foi adequada, uma vez que, pelo teste de envelhecimento artificial, foi possível obter sementes com vigor variando de $78 \%$ a $11,5 \%$.

Verifica-se que não houve diferença significativa entre os valores de condutividade elétrica, entre os diferentes tratamentos. $\mathrm{O}$ resultado esperado seria que, quanto maior o tempo de envelhecimento das sementes, maior deveria ser o nível de condutividade elétrica. Embora a tendência esperada fosse esta, o maior tempo de envelhecimento (lote 5) não correspondeu ao maior nível de condutividade elétrica. Não houve diferenciação consistente dos lotes ao longo do período experimental, pois os mesmos apresentaram comportamentos variáveis, ora registrando valores mais altos, ora mais baixos. Uma explicação para esta variação, possivelmente, resida no fato deste teste ter sido conduzido imediatamente após o envelhecimento acelerado, com as sementes ainda úmidas. Nesta condição, provavelmente, as membranas se reorganizaram durante $\mathrm{o}$ teste, retomando a sua seletividade.

Examinando-se as médias obtidas no teste de germinação em sementes expostas em diferentes períodos de envelhecimento artificial, verifica-se que as sementes envelhecidas por um período de 96 horas (lote 3) apresentaram germinação de $82 \%$, não diferindo estatisticamente das sementes que não passaram pelo envelhecimento artificial (lote 1) e das que foram submetidas ao envelhecimento por 192 horas (Quadro 2).

Quanto ao teste de envelhecimento acelerado, quanto maior o tempo de exposição das sementes ao envelhecimento artificial, menores foram os valores de vigor. Observa-se que o envelhecimento acelerado foi mais sensível em detectar a deterioração das sementes envelhecidas que os testes de condutividade elétrica e germinação. Estes resultados conferem com os observados por Rodrigues et al. (1995), que compararam vários testes de vigor na avaliação da qualidade de sementes de feijãode-vagem, estando o teste de envelhecimento acelerado entre os que apresentaram os melhores resultados na avaliação do potencial de emergência das plântulas em campo.

QUADRO 2 - Médias obtidas nos testes de germinação, vigor e acidez graxa em sementes envelhecidas artificialmente.

\begin{tabular}{|c|c|c|c|c|c|c|c|c|}
\hline \multirow{3}{*}{$\begin{array}{c}\text { Lotes } \\
1\end{array}$} & \multicolumn{8}{|c|}{ TESTE DE QUALIDADE } \\
\hline & \multicolumn{2}{|c|}{$\begin{array}{c}\text { Condutividade } \\
\text { elétrica }\left(\mu S \mathrm{~cm}^{-1} \mathrm{~g}^{-1}\right)\end{array}$} & \multicolumn{2}{|c|}{ Germinação (\%) } & \multicolumn{2}{|c|}{$\begin{array}{l}\text { Envelhecimento } \\
\text { acelerado (\% de } \\
\text { germinação) }\end{array}$} & \multicolumn{2}{|c|}{$\begin{array}{r}\text { Acidez graxa (mL } \\
\text { de } \mathrm{KOH} / 100 \mathrm{~g} \mathrm{MS})\end{array}$} \\
\hline & 37,14 & $\mathrm{~A}$ & 88,50 & A & 78,00 & $\mathrm{~A}$ & 4,19 & A \\
\hline 2 & 31,89 & A & 85,00 & $\mathrm{AB}$ & 37,50 & B & 4,68 & B \\
\hline 3 & 29,16 & A & 82,00 & $\mathrm{ABC}$ & 34,00 & $\mathrm{BC}$ & 5,04 & $\mathrm{BC}$ \\
\hline 4 & 35,02 & A & 71,00 & $\mathrm{BC}$ & 18,50 & $\mathrm{BC}$ & 5,35 & $\mathrm{C}$ \\
\hline 5 & 34,78 & A & 70,00 & $\mathrm{C}$ & 11,50 & $\mathrm{C}$ & 6,70 & D \\
\hline $\mathrm{CV}(\%)$ & 12 & & 8 & & 29 & & 3 & \\
\hline
\end{tabular}

Médias de tratamentos seguidas de pelo menos uma letra igual, na coluna, não diferem significativamente ( $>0,05)$.

Ciênc. agrotec., Lavras, v. 30, n. 4, p. 679-684, jul./ago., 2006 
Vieira et al. (1999) concluíram que o envelhecimento artificial apresentou-se como sendo um teste de vigor eficiente na determinação da qualidade fisiológica de sementes de sorgo. Também Menten (1978), estudando o teste de vigor em sementes de feijão, obteve correlação significativa da primeira contagem, emergência e envelhecimento acelerado; isto indica que eles podem ser aplicados indistintamente na detecção de níveis de vigor, porém, o teste de envelhecimento acelerado foi o que apresentou maior amplitude de variação nos resultados, sendo, portanto, mais sensível.

O teste de ácidos graxos livres apresentou a maior amplitude de variação nos resultados, diferenciando-se entre os demais testes por se mostrar mais sensível para avaliar a qualidade fisiológica de sementes de arroz, podendo-se observar uma maior estratificação entre os lotes (Quadro 2). Resultados semelhantes foram obtidos por Soares et al. (2001) ao avaliar o teste de ácidos graxos livres como teste de qualidade em sementes de milho armazenadas. $\mathrm{O}$ autor concluiu que este apresentou-se mais eficiente para detectar alterações na qualidade da semente armazenada por seis meses que o teste padrão de germinação, cujos valores não apresentaram diferenças significativas ao longo do tempo de armazenamento. Biaggioni \& Ferreira (1998) relataram que, durante o armazenamento, a hidrólise do material graxo iniciase antes da hidrólise de proteínas ou carboidratos; por isso, o teor de ácidos graxos livres pode ser usado como um indicador da deterioração dos grãos.

Os resultados da análise de correlação entre o teste de acidez graxa e os de germinação e vigor são apresentados no Quadro 3. Verifica-se que o teste de acidez graxa correlacionou-se significativamente com os testes de germinação e envelhecimento acelerado, apresentando os coeficientes de correlação de $63 \%$ e $73 \%$, respectivamente, sendo estes negativo, ou seja, à medida que aumentam os valores obtidos no teste de ácidos graxos livres no decorrer do tempo de envelhecimento artificial, diminuem os valores de germinação e envelhecimento acelerado. Já a correlação entre acidez graxa e condutividade elétrica não foi significativa, com o coeficiente de correlação de 10\%. Embora os valores de condutividade elétrica devessem aumentar ao longo do período de envelhecimento das sementes, correlacionando-se, assim, significativamente com o teste de acidez graxa, esse não foi o resultado obtido nas análises realizadas, descritas anteriormente, o que explica a correlação não significativa entre os referidos testes.

\section{Acidez graxa e classificação comercial em grãos de arroz}

No Quadro 4 estão apresentados os resultados provenientes das análises de acidez graxa realizadas nos cinco tipos comerciais de grãos de arroz.

Verifica-se, pela análise do Quadro, que as médias dos valores obtidos no teste de ácidos graxos livres diferiram estatisticamente, podendo-se observar a estratificação consistente dos cinco tipos de arroz. Podese observar a tendência do nível de ácidos graxos livres acompanhar a classificação comercial por tipos, conforme o esperado. Assim, quanto pior o tipo comercial de grãos de arroz (5), maior o nível de ácidos graxos livres $(4,84 \mathrm{~mL}$ $\mathrm{KOH} / 100 \mathrm{~g} \mathrm{MS}$ ), confirmando a sensibilidade do teste.

QUADRO3 - Resultados fornecidos pela análise de correlação entre acidez graxa e os demais testes de qualidade de sementes.

\begin{tabular}{|cccc|}
\hline Teste & $\mathbf{N}^{\mathbf{0}}$ de Observações & Coeficiente de Correlação & Significância \\
\hline Germinação & 20 & $-0,6333$ & Significativo \\
Envelhecimento Acelerado & 20 & $-0,7326$ & Significativo \\
Condutividade Elétrica & 20 & 0,1011 & não signific. \\
\hline
\end{tabular}

QUADRO 4 - Nível de ácidos graxos livres encontrados em cinco tipos comerciais de grãos de arroz:

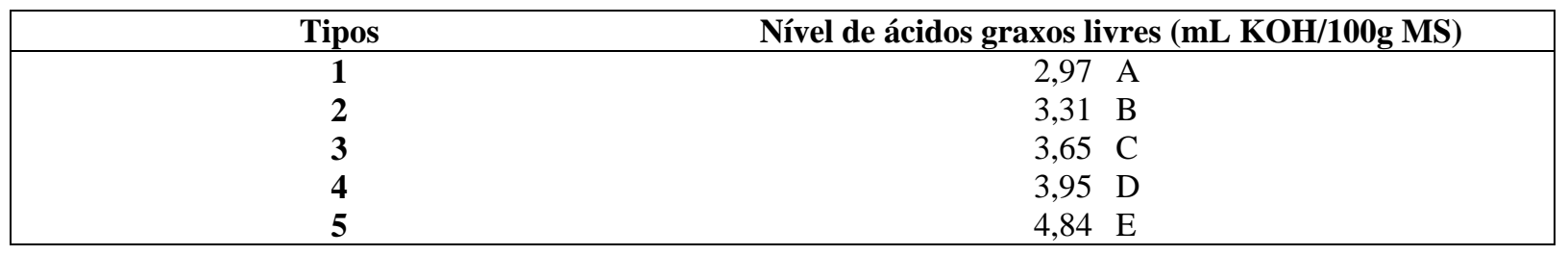

Médias de tratamentos seguidas de pelo menos uma letra igual, não diferem significativamente $(\mathrm{P}>0,05)$. 


\section{CONCLUSÕES}

Com base nos resultados obtidos nos testes experimentais, pôde-se obter as seguintes conclusões:

- o teste de acidez graxa é promissor para avaliação do vigor em sementes de arroz;

- o nível de ácidos graxos livres está relacionado à classificação comercial por tipos, em grãos de arroz.

\section{REFERÊNCIAS BIBLIOGRÁFICAS}

ABDUL-BAKI, A. A.; ANDERSON, J. V. Physiological and biochemical deterioration of seeds. In: KOZLOWSKI, T. T. (Ed.). Seed biology. 2. ed. New York: Academic, 1972. p. 283-316.

AMERICAN ASSOCIATION OF CEREAL CHEMISTS. AACC methods 02-02A: fat acidity: rapid method, for grain. In: Approved methods of the American Association of the Cereal Chemists. Saint Paul, 1995. v. 1.

BIAGGIONI, M. A. M.; FERREIRA, W. A. Variação na germinação e nível de ácidos graxos livres durante o armazenamento de milho colhido mecanicamente. In: CONGRESSO BRASILEIRO DE ENGENHARIA AGRÍCOLA, 27., 1998, Poços de Caldas. Anais... Lavras: UFLA/SBEA, 1998.

BRASIL. Ministério da Agricultura e Reforma Agrária. Regras para análise de sementes. Brasília, DF: SNDA/ DNDV/CLAV, 1992.364 p.

KRZYZANOWSKI, F. C.; VIEIRA, R. D.; FRANÇA NETO, J. B. Vigor de sementes: conceitos e testes. Londrina: ABRATES, 1999. 218 p.

MARCOS FILHO, J.; CÍCERO, S. M.; SILVA, W. R. Avaliação da qualidade das sementes. Piracicaba: FEALQ, 1987. 230 p.

MENTEN, J. O. M. Sanidade, germinação e vigor de sementes de feijão (Phaseolus vulgaris L.). Summa Phytopathologica, Piracicaba, v. 4, n. 2/4, p. 105-110, 1978.

PEREIRA, P. A. Alterações bioquímicas e germinação de sementes de paspalum paniculatum armazenadas sob diferentes condições. 1999. Dissertação (Mestrado) Universidade Federal de Lavras, Lavras, 1999.

RIBEIRO, D. M. C. A. Adequação do teste de condutividade elétrica de massa e individual para avaliação da qualidade fisiológica de sementes de milho (Zea mays $\boldsymbol{L}$.). 1999. 105 p. Tese (Doutorado em Fitotecnia) - Universidade Federal de Lavras, Lavras, 1999.

RODRIGUES, V. L. F.; ALVARENGA, E. M.; SILVA, R. F.; CARDOSO, A. A. Comparação de testes para a avaliação da qualidade de sementes de feijão-de-vagem. In: CONGRESSO BRASILEIRO DE SEMENTES, 9., 1955, Florianópolis. Informativo ABRATES, Londrina, v. 5, n. 2, p. 132, 1995.

SOARES, T. A. Análise da acidez graxa como índice de qualidade em grãos de soja. $74 \mathrm{f}$. Dissertação (Mestrado em Agronomia) - Universidade Estadual Paulista, Botucatu, 2003.

SOARES, T. A.; BIAGGIONI, M. A. M.; PASSOS, R. S. Comparação entre os testes de acidez graxa e germinação como índices de qualidade em sementes de milho armazenado. In: CONGRESSO BRASILEIRO DE ENGENHARIA AGRÍCOLA, 27., 2001, Foz do Iguaçu. Anais... Foz do Iguaçu: SBEA, 2001.

VIEIRA, R. D. Teste de condutividade elétrica. In: VIEIRA, R. D.; CARVALHO, N. M. (Eds.). Testes de vigor em sementes. Jaboticabal: FUNEP, 1994. p. 103-132.

VIEIRA, E. S. N.; PINHO, E. V. R. von; RESENDE, M. L. Avaliação de métodos de colheita e qualidade fisiológica e sanitária de sementes de sorgo. Revista Brasileira de Armazenamento, [S.1.], v. 21, n. 2, p. 249254, 1999.

YANAGIWARA, R. S.; BIAGGIONI, M. A. M.; CAVARIANI, C.; MARTINS, C. Análise de acidez graxa em sementes de trigo. In: CONGRESSO BRASILEIRO DE ENGENHARIA AGRÍCOLA, 33., 2004, São Pedro. Anais... Campinas: UNICAMP/EMBRAPA/SBEA, 2004. 